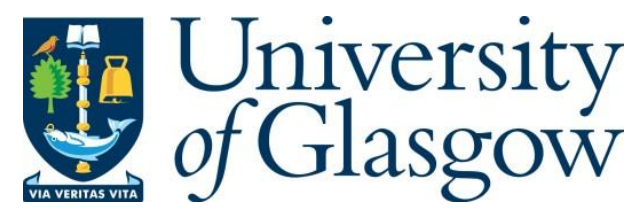

Zhang, J., Akinsolu, M. O., Liu, B. and Zhang, S. (2021) Design of zero clearance SIW endfire antenna array using machine learning-assisted optimization. IEEE Transactions on Antennas and Propagation, (doi: 10.1109/TAP.2021.3137500).

There may be differences between this version and the published version. You are advised to consult the publisher's version if you wish to cite from it.

https://eprints.gla.ac.uk/257701/

Deposited on: 25 October 2021

Enlighten - Research publications by members of the University of Glasgow https://eprints.gla.ac.uk 


\title{
Communication Design of Zero Clearance SIW Endfire Antenna Array Using Machine Learning-Assisted Optimization
}

\author{
Jin Zhang, Mobayode O. Akinsolu Member, IEEE, Bo Liu Senior Member, IEEE, and Shuai Zhang Senior Member, IEEE
}

\begin{abstract}
In this paper, a substrate integrated waveguide (SIW) endfire antenna array with zero clearance is proposed for 5 th generation (5G) mobile applications using machine learning-assisted optimization. In particular, a novel impedance matching architecture that involves three arbitrary pad-loading metallic vias is investigated and adopted for the antenna element. Due to the stringent design requirements, the locations and sizes of the vias and pads are obtained via a state-ofthe-art machine learning assisted antenna design exploration method, parallel surrogate model-assisted hybrid differential evolution for antenna synthesis (PSADEA). Keeping a very low profile, the array optimized by PSADEA covers an operating frequency bandwidth from $36 \mathrm{GHz}$ to 40 GHz. The in-band total efficiency is generally better than $60 \%$ and the peak gain is above $5 \mathrm{dBi}$. The beam scanning range at $39 \mathrm{GHz}$ covers from $-20^{\circ}$ to $35^{\circ}$.
\end{abstract}

Index Terms-Design exploration, SIW endfire antenna, optimization, antenna array, surrogate modelling.

\section{INTRODUCTION}

Beam-steerable arrays are widely used for 5G millimeter-wave (mm-wave) applications (e.g., handset devices) to achieve both large spatial coverage and high gain [1]. Due to an increasing demand for thinner profiles and larger screens in the design of present-day mobile devices, there is a requirement to reduce antenna profile and clearance for better integration with the chip set and the printed circuit board (PCB) of handset devices. Consequently, the clearance, which is the area to be reserved on the metal ground plane to guarantee the antenna's proper functioning, becomes an important measure for $5 \mathrm{G}$ mobile antenna design. Antennas that have zero clearance with the capability of being totally integrated with the PCB board circumvent this bottle neck.

Many works have presented the design of 5G mm-wave antennas having small clearance. For example, in [2], a quasi-yagi antenna array operating at $28 \mathrm{GHz}$ with $2.5 \mathrm{~mm}\left(0.23 \lambda_{0}\right.$, where $\lambda_{0}$ is the wavelength in free space) clearance was proposed, and in [3], the clearance of a proposed dipole array is reduced to $1.2 \mathrm{~mm}\left(0.15 \lambda_{0}\right)$. In [4], a dual-polarized SIW/dipole array is proposed with a clearance of $0.16 \lambda_{0}$. However, it is very challenging to reduce the clearance of dipole antennas to zero by covering them with closely placed metal plates due to the deterioration of the radiation efficiency and impedance matching.

In contrast to dipole antennas and some other antenna types, substrate integrated waveguide (SIW) endfire antennas are excellent candidates for mobile applications where antennas need to be highly integrated with PCBs. Even though SIW open-ended antennas are well known as low profile antennas, they suffer from significant reflection at the waveguide-to-air interface when the thickness of the PCB is reduced to approximately $0.1 \lambda_{0}$ [5]. To address this bottleneck, many methods have been proposed for impedance matching and

J. Zhang and S. Zhang (corresponding author: sz@es.aau.dk) are with the Antennas, Propagation and Millimeter-wave Systems, Department of Electronic Systems, Aalborg University, 9220 Aalborg Ø, Denmark.

Mobayode O. Akinsolu (mobayode.akinsolu@glyndwr.ac.uk) is with Faculty of Arts, Science and Technology, Wrexham Glyndŵr University, Wrexham LL11 2AW, UK.

Bo Liu (bo.liu@glasgow.ac.uk) is with James Watt School of Engineering, University of Glasgow, Glasgow G12 8QQ, UK.

to broaden the bandwidth. Popular approaches include loading with parallel transition plates [5], [6], dielectric lens [7], metamaterials [8], or the combination of different techniques [9]. For all the above mentioned solutions, enhancing the bandwidth comes with a cost of enlarging the clearance since the matching structures are loaded outside the opening end of the SIW.

SIW antennas with zero clearance and planar structures have been proposed, such as in [10] and [11]. In [10], the H-plane horn antenna employs a tapered metallic ridge inside the waveguide to achieve a wideband impedance matching. In [11], the SIW H-plane horn antenna achieved high efficiency and narrow band matching by using an air-filled waveguide and a tapered microstrip feeding line. A noticeable drawback with these approaches is the large opening angle of the waveguide, which has a great contribution to the impedance matching but also limits its application when considering beamsteering.

In this paper, a zero clearance and low profile SIW endfire array is proposed by implementing a novel impedance matching architecture. The proposed structure includes three arbitrary blind vias inside the SIW, which provides three resonances to cover the required operating band. Due to the sensitive response of the interference between each via (see Section II), the locations and sizes of the vias are critical considering the desired performances for bandwidth, realized gain, and total efficiency. Given that the conventional trialand-error, parameter sweeping, and local optimization methods are infeasible, global optimization methods are needed to find the best set of geometric parameters that meet the desired performance.

A global optimization algorithm which is widely used in antenna design exploration, the particle swarm optimization (PSO), was employed through Computer Simulation Technology Microwave Studio (CST-MWS), but satisfactory design solutions were not obtained (see Section III). Hence, a state-of-the-art machine learningassisted antenna design exploration method, the parallel surrogate model-assisted hybrid differential evolution for antenna synthesis (PSADEA) [12], [13], is then employed. Among related state-ofthe-art methods with various kinds of advantages, e.g., [14]-[16], PSADEA carries out single objective constraint global optimization and has the characteristics of high optimization ability, no need for an initial design, and can handle antenna cases with more than 30 design variables. Compared to standard global optimization methods (e.g., PSO, differential evolution (DE), genetic algorithm), it can obtain design solutions with higher quality and with over 20 times optimization speed improvement [12], [13]. The PSADEA-optimized SIW antenna shows satisfactory performance in terms of bandwidth, realized gain, and total efficiency, for both simulated and measured results.

The remainder of this paper is organized as follows: Section II provides the design guidelines and modus operandi of the proposed SIW endfire antenna, Section III details the optimization procedure, Section IV discusses the proposed antenna's performance (simulated and measured) and the concluding remarks are given in Section V. 


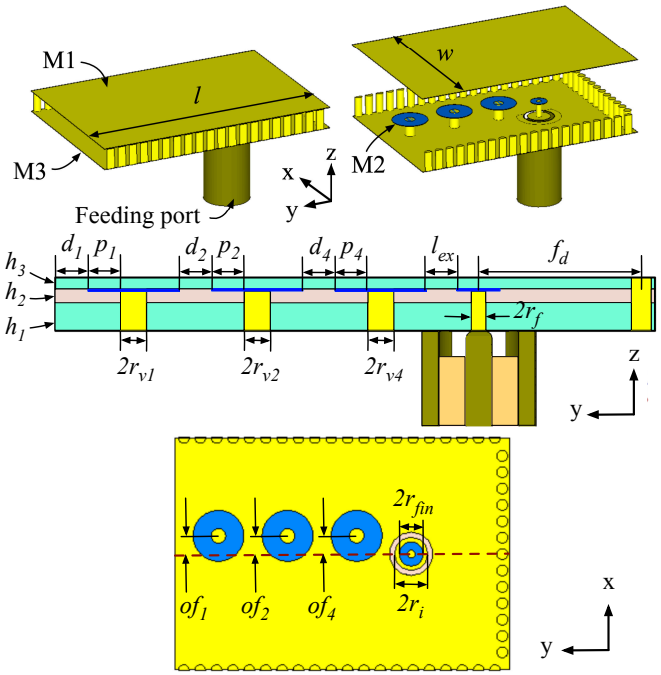

Fig. 1: Antenna element configuration.

\section{Antenna Working PRINCIPLe}

The SIW endfire antenna was modeled and discretized in CSTMWS using a cell density of 15 cells per wavelength to have about $1,850,000$ hexahedral mesh cells in total for optimizations. The model is analyzed using time-domain finite integration technique (FIT) with an accuracy of $-40 \mathrm{~dB}$, and each full-wave EM simulation costs about 20 minutes (from a wall clock) on average on a workstation with an Intel 8-core i9-9900K 3.6 GHz CPU, 64 GB RAM and 8 GB NVIDIA Quadro RTX 4000 GPU. The configuration of the initial model is shown in Fig. 1. The PCB consists of three substrate layers. The top and bottom layers are both RO4350B having a dielectric constant $\left(\epsilon_{r}\right)$ of 3.66 and a loss tangent $(\tan \delta)$ of 0.0037 . The middle layer is RO4450F with $\epsilon_{r}$ of 3.7 and $\tan \delta$ of 0.004 . The thicknesses of the top, middle, and bottom layers are $h_{1}, h_{2}$, and $h_{3}$, respectively. Hence, the total thickness of the PCB is $\left(h_{1}+h_{2}+h_{3}\right)$. The ground plane, which are the top and bottom copper metal surfaces of the SIW, are labelled as $M 1$ and $M 3$, respectively. The height of each of the vias is $h_{1}+h_{2}$. One end of each of the vias is grounded by a connection to $M 3$ and the other end of each of the vias is loaded with a round pad marking as an additional copper layer (labelled as $M 2$ in Fig. 1).

The transmission line (TL) model of the SIW antenna, loaded with multiple vias is shown in Fig. 2. $Z_{S I W}$ and $R_{a}$ represent the characteristic impedance of the SIW and the radiation resistance in the free space, respectively. By introducing vias inside the SIW, multiple resonances can be generated. Each via can be seen as a series of a inductance $L_{p n}(n=1,2, \ldots)$ and a capacitance $C_{v n}$, which determines the resonant frequency. The value of $L_{p n}$ and $C_{v n}$ is directly controlled by the diameter and height of the corresponding via $\left(r_{v n}\right.$ and $\left.h_{1}+h_{2}\right)$, and the size of the pad $\left(p_{n}\right)$ on this via. Besides, the coupling capacitance between the adjacent vias $\left(C_{p n}\right)$ has noticeable influences on the resonant frequencies and bandwidth as well. The coupling can be tuned by varying the distance $\left(d_{n}\right)$ between the vias. Another sensitive parameter is the location of the vias relative to the SIW. Hence, all the vias are located close to the center line of the SIW with a small offset $\left(o f_{n}\right)$.

The TL model can be verified by the performances of a simple onevia model (Model-A) and a two-via model (Model-B). The structure inside the SIW of Model-A and Model-B are shown in Fig. 3(a) and Fig. 3(b), respectively. In order to further simplify the model for simulation, the substrate is reduced to a single layer of RO4350B

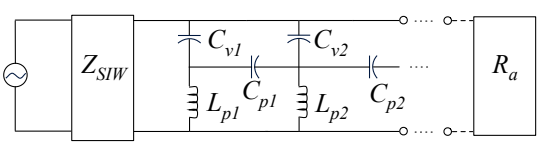

Fig. 2: TL model of multiple vias loaded SIW antenna.

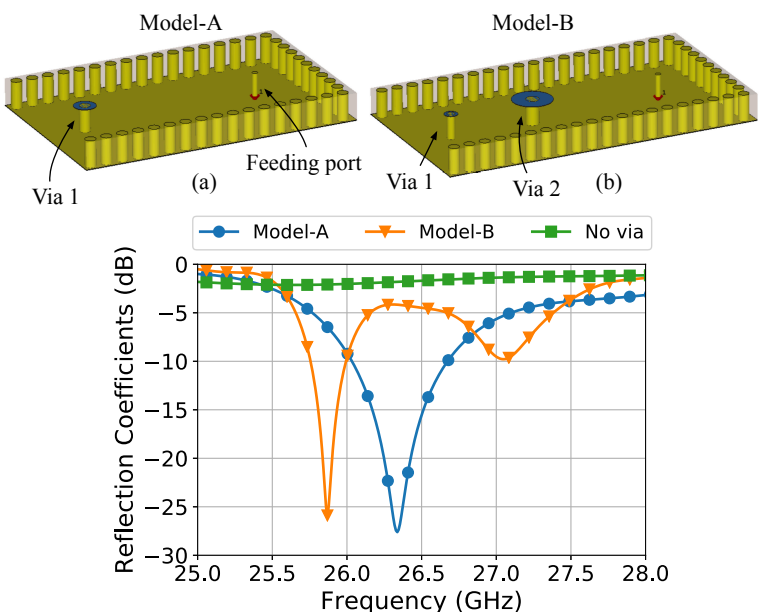

Fig. 3: Simplified antenna models and reflection coefficients (a) Model-A with one via loaded. (b) Model-B with two vias loaded. (c) reflection coefficients.

with a thickness of $1 \mathrm{~mm}$ and the height of the vias are fixed at 0.9 mm. Fig. 3(c) presents the reflection coefficients of Model-A, modelB, and a comparison SIW antenna without loading. From Fig. 3(c), it can be seen that without any impedance matching structure, the SIW antenna is totally mismatched at the observed frequency band. By adding one via (Via 1) close to the SIW opening end, ModelA obtains one passband at the resonant frequency of $26.3 \mathrm{GHz}$. When another via (Via 2) is added between Via 1 and the feeding port, Model-B obtains two resonances at $25.8 \mathrm{GHz}$ and $27 \mathrm{GHz}$, respectively. The antenna quality factor $(\mathrm{Q})$ can be estimated by (1), where $f_{c}$ is the center frequency, $f_{1}$ and $f_{2}$ are the cut-off frequencies of the lower and higher bound.

$$
Q_{A}=f_{c} /\left(f_{2}-f_{1}\right)
$$

If the return loss of $6 \mathrm{~dB}$ is taken as a reference for defining the frequency span, the $\mathrm{Q}$ value of Model- $\mathrm{A}$ is 23.5 , whereas the $\mathrm{Q}$ values of the two resonances of Model-B are 61.9 and 51.6, respectively. Apparently, the two resonances in Model-B have a higher Q, which results in a narrower bandwidth each. It gives Model-B the potential to serve as a dual-band antenna or a single band antenna by tuning the two resonances closer. Apart from the dimensions and the locations of the vias, the resonant frequencies and $\mathrm{Q}$ factors are also influenced by other parameters such as the width of SIW, the PCB thickness, and the substrate characteristics.

In Model-A, the resonant frequency can be easily tuned by changing the diameter of Via 1 and the size of the pad. In Model-B, Via 1 mainly affects lower resonance. As shown in Fig. 4(a), the lower resonant frequency moves higher when the diameter of Via $1 r_{v 1}$ is larger, which corresponds to a lower inductance. Meanwhile, the higher resonant frequency does not move. The influence of Via 2 is more complicated because it affects both resonances. As shown in Fig. 4(b), when the diameter of Via $2\left(r_{v 2}\right)$ is larger, both resonant frequencies move higher. A similar phenomenon is observed from the locations of the vias as well. In Fig. 4(c), the location of Via $1\left(d_{1}\right)$ 


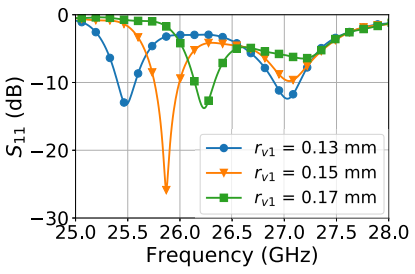

(a)

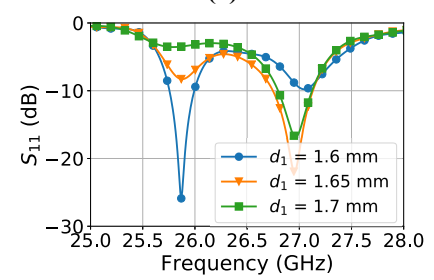

(c)

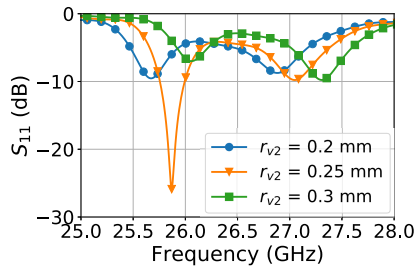

(b)

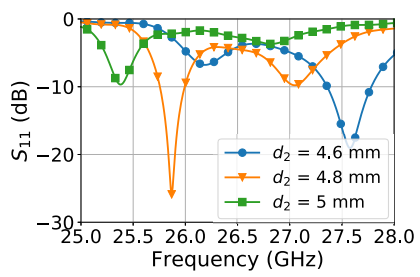

(d)

Fig. 4: Reflection coefficients of Model-B with different via diameters and locations (a) Diameter of Via 1. (b) Diameter of Via 2. (c) Distance from Via 1 to the SIW opening edge. (d) Distance from Via 2 to Via 1.

influences the impedance matching but does not shift the resonant frequencies. However, in Fig. 4(d), both impedance matching and resonant frequencies change with the location of Via $2\left(d_{2}\right)$. It is reasonable that Via 2 is more sensitive than Via 1, since it involves both the coupling to Via 1 and the feeding port. However, this causes great difficulties when more vias are applied in order to achieve wider bandwidth by introducing more resonances, since it is very difficult to find a pattern among a lot of sensitive parameters by manual tuning or parameter sweeping. Hence, design exploration via machine learningassisted global optimization is carried out in the next Section.

\section{MACHINE LEARNING-ASSISTED DESIGN EXPLORATION}

In order to achieve larger bandwidth for the proposed SIW antenna, one possible solution is to add more vias inside the SIW. However, the influences among multiple vias are complicated. Specifically, each resonance is influenced by a large number of design parameters (i.e., more than 20) and each parameter could influence several resonances as well. The high level of interrelation between the design parameters makes the conventional experience-driven trial-and-error method and parameter sweeping method infeasible.

Using local optimization (e.g., CST TRF) from an initial design is a popular method [17], [18]. However, this is also infeasible for this SIW antenna. Because the amount of inductance and capacitance introduced by a metallic via is difficult to ascertain, an initial design with reasonably good quality is difficult to be derived or estimated. Without a good initial design, local optimization methods are less likely to obtain a satisfactory design. To verify this, TRF in CSTMWS is used from three initial designs obtained by Latin hypercube sampling [19] of the design space. Although a sigma value of unity is used to avoid being trapped in a local optimum, the obtained designs are still trapped in local optima, whose performances are far from satisfactory (Table I shows the best design obtained in three runs). This shows the necessity of employing artificial intelligence (AI) techniques (i.e., machine learning) for design exploration.

\section{A. Antenna element design exploration}

Global optimization is applied on the three vias model shown in Fig. 1. The critical design parameters and their ranges and the geometric constraints described in Table II have been considered.
The optimization goal is to achieve the maximum bandwidth in the frequency spectrum of $24 \mathrm{GHz}$ to $40 \mathrm{GHz}$, where the maximum inband reflection coefficient is less than or equal to $-10 \mathrm{~dB}$, subject to a minimum in-band realized gain better than $0 \mathrm{dBi}$ and a minimum in-band total efficiency better than or equal to $60 \%$. The optimization has been implemented on the workstation described in Section II. The time reported is from a wall clock.

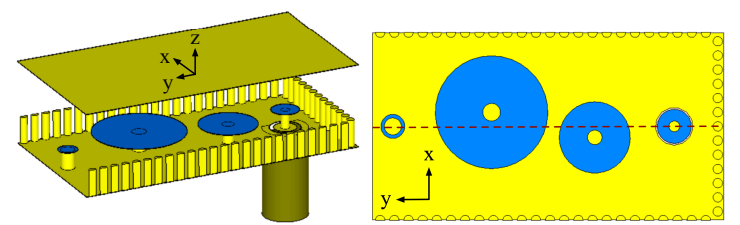

(a)

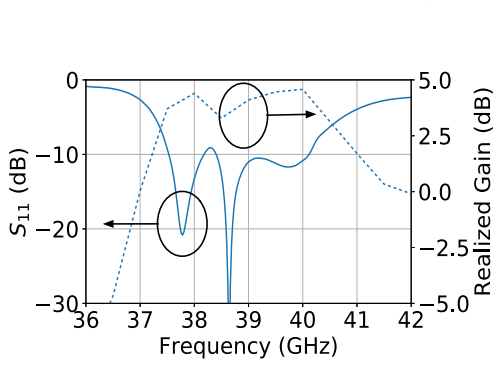

(b)

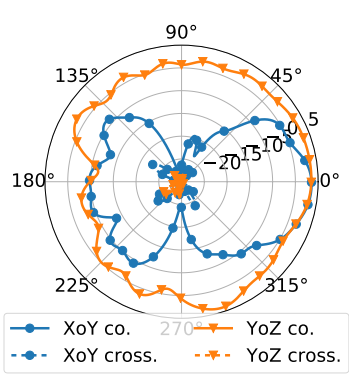

(c)
Fig. 5: Antenna element configuration and performances. (a) Antenna configuration. (b) $S_{11}$ and realized gain. (c) Radiation pattern at 39 $\mathrm{GHz}$.

PSO [20] is a metaheuristic algorithm based on the concept of swarm intelligence, which is widely used in antenna design exploration (e.g., [21]). Here, PSO in CST-MWS is used with a swarm size of 50, a computing budget of 2,000 EM simulations; all other algorithmic settings are the default settings in CST-MWS. Since each run of PSO costs about one month, three runs are carried out. All of them failed to obtain satisfactory designs, as shown in Table I for their typical results (the best design over 3 runs). This shows the necessity of introducing advanced machine learning-assisted global optimization methods.

PSADEA [12], [13] is one of the state-of-the-art machine learningassisted global optimization methods considering the design landscape characteristics of antennas and arrays. It employs Gaussian process supervised learning and differential evolution (DE) to predict antenna performances and conduct global optimization, respectively. Compared to other state-of-the-art methods in the SADEA series [22], it aims at satisfying stringent design specifications for antennas with less than 30 design parameters. To achieve this, the cooperative use of complementary DE mutation operators, which is controlled by a reinforcement learning scheme, is the main driving force; an adaptive Gaussian process supervised learning scheme supporting the above search scheme is also essential. More details about PSADEA can be found in [12], [13].

In PSADEA-based design exploration, a population size of 50 is used (same as CST-MWS PSO) and the computing budget is $500 \mathrm{EM}$ simulations. All other PSADEA settings are the default settings in [12], [13]. The convergence criterion is an improvement of less than $1 \mathrm{e}-3$ in the objective value after $100 \mathrm{EM}$ simulations. After 5 days' optimization, PSADEA obtains the satisfactory design in Table II using 312 EM simulations. In comparison to the best designs obtained by the CST-MWS optimizers, the PSADEA-optimized design offers $2.1 \mathrm{GHz}$ and $2.2 \mathrm{GHz}$ more bandwidth compared to the best designs 
obtained by CST TRF and CST PSO, respectively. The satisfactory performance of the PSADEA-optimized design in terms of bandwidth, realized gain and radiation efficiency ensured the viability of the proposed SIW endfire antenna for physical implementation (i.e., prototyping and fabrication).

Fig. 5(a) demonstrates the proposed antenna structure obtained from the optimization. Fig. 5(b) shows the $S_{11}$ and realized gain of the proposed model. The best bandwidth is obtained from 37.5 $\mathrm{GHz}$ to $40.1 \mathrm{GHz}$, where the realized gain within the bandwidth is above $3.3 \mathrm{dBi}$. The radiation patterns are stable over the operating band. As an example, the realized gain at $39 \mathrm{GHz}$ is $4.1 \mathrm{dBi}$ and the radiation pattern is shown in Fig. 5(c). The cross-polarization is below $-20 \mathrm{~dB}$ in the radiation direction for all frequencies.

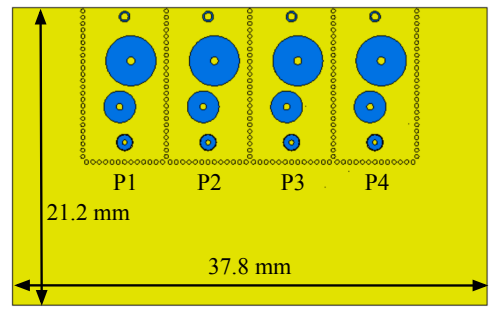

Fig. 6: 4-element array configuration. (a)

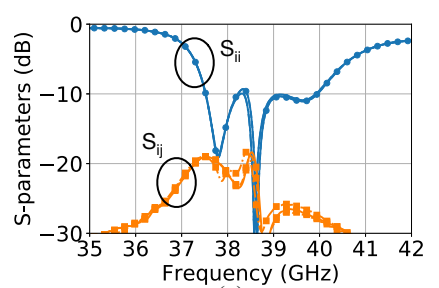

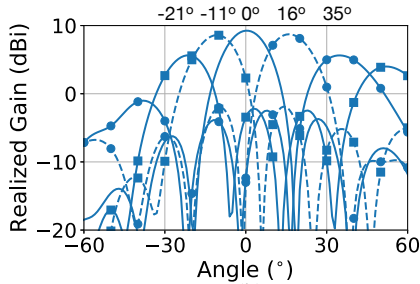

(b)
Fig. 7: Array performances. (a) Reflection coefficients and mutual coupling. (i,j $=\{1,2,3,4\}$.) (b) Beam scanning pattern at $39 \mathrm{GHz}$.

It is worth noting that further increasing the number of vias can stimulate more resonances but the benefit on the bandwidth is limited. For one thing, it is because the $\mathrm{Q}$ value of each resonance increases and for another thing, the interference between the vias prevents some of the resonances to form a wide band. As an example, the best bandwidth of a model with five vias is $7.2 \%$, which has little advantage compared to the proposed model with three vias considering the complexity of the structure.

\section{B. Array performances}

The proposed antenna is applied in a 4-element array. The inside configuration is shown in Fig. 6. The ground plane size of the array is $21.2 \mathrm{~mm} \times 37.8 \mathrm{~mm}$. Fig. 7(a) presents the reflection coefficients of all the four array elements and the mutual coupling between the adjacent elements. The array elements maintains the same bandwidth as the single antenna from $37.5 \mathrm{GHz}$ to $40 \mathrm{GHz}$ and the mutual coupling is below $-18 \mathrm{~dB}$ within the bandwidth. Fig. 7(b) shows the beam scanning pattern at $39 \mathrm{GHz}$. The scanning range is from $-20^{\circ}$ to $35^{\circ}$ and the realized gain ranges from $5.62 \mathrm{dBi}$ to $9.25 \mathrm{dBi}$.

\section{Measurements And Results}

The proposed antenna and the 4-element array are fabricated and measured. The front and back photographs of the fabricated single antenna and array are shown in Fig. 8. The antennas are fed with MMPX connectors mounted on the back side of the PCB. The antenna
TABLE I: (Previous Table II) Optimization results of the synthesized SIW Endfire Antenna (PSADEA, TRF, PSO).

\begin{tabular}{lcccc}
\hline Results & $\begin{array}{r}\text { PENAlty } \\
\text { WEIGHT }\end{array}$ & PSADEA & TRF & PSO \\
\hline Bandwidth (GHz) & 1 & $37.5-40.0$ & $27.3-27.7$ & $35.2-35.5$ \\
\hline $\begin{array}{l}\text { Minimum In-Band } \\
\text { Realized Gain (dBi) }\end{array}$ & 50 & 3.3 & 2.7 & 2.6 \\
\hline $\begin{array}{l}\text { Minimum In-Band } \\
\text { Total Efficiency }\end{array}$ & 50 & $60 \%$ & $72 \%$ & $55 \%$ \\
\hline
\end{tabular}

TABLE II: (Previous Table I) Search space for the structure parameters and optimal values obtained by PSADEA (units in $\mathrm{mm}$ ). All parameters are continuous variables, with the exceptions of $h_{1}$ and $h_{3}$, which take values in $\{0.422,0.508,0.762\}$ and $\{0.101,0.168,0.254,0.338,0.422,0.508\}$, respectively. $h_{1}$ and $h_{3}$ must satisfy the constraint $h_{1}+h_{2}+h_{3} \leq 1.2 . r_{i}$ and $r_{f}$ must satisfy the constraint $r_{f} \leq r_{i}-0.1$.

\begin{tabular}{lccc}
\hline DESCRIPTION & PARAM. & VALUES & OPTIMUM \\
\hline SPACING: VIA 1 / OPENING & $d_{1}$ & {$[0.0,1.5]$} & 0.27 \\
SPACING: VIA 2 / VIA 1 & $d_{2}$ & {$[0.0,3.0]$} & 0.97 \\
SPACING: VIA 3 / VIA 2 & $d_{3}$ & {$[0.0,7.0]$} & 0.36 \\
SPACING: FEED / END & $f_{d}$ & {$[1.5,3.0]$} & 1.58 \\
SPACING: VIA 3 / FEED & $l_{e x}$ & {$[0.0,4.0]$} & 1.16 \\
OFFSET: VIA 1 FROM CENTER & $o f_{1}$ & {$[-0.5,0.5]$} & 0.00 \\
OFFSET: VIA 2 FROM CENTER & $o f_{2}$ & {$[-0.5,0.5]$} & 0.45 \\
OFFSET: VIA 3 FROM CENTER & $o f_{3}$ & {$[-0.5,0.5]$} & -0.35 \\
PAD WIDTH OF VIA 1 & $p_{1}$ & {$[0.0,1.0]$} & 0.12 \\
PAD WIDTH OF VIA 2 & $p_{2}$ & {$[0.0,2.0]$} & 1.51 \\
PAD WIDTH OF VIA 3 & $p_{3}$ & {$[0.0,2.0]$} & 0.90 \\
DIAMETER: SOLDER PAD & $r_{i}$ & {$[0.3,0.5]$} & 0.45 \\
DIAMETER: FEED PIN & $r_{f}$ & {$[0.1,0.3]$} & 0.17 \\
DIAMETER: FEED PAD & $r_{f i n}$ & {$[0.0,0.5]$} & 0.37 \\
DIAMETER: VIA 1 & $r_{v 1}$ & {$[0.1,0.5]$} & 0.25 \\
DIAMETER: VIA 2 & $r_{v 2}$ & {$[0.1,0.5]$} & 0.25 \\
DIAMETER: VIA 3 & $r_{v 3}$ & {$[0.1,0.5]$} & 0.23 \\
SIW WIDTH & $w$ & {$[5.4,6.6]$} & 5.94 \\
THICKNESS: BOTTOM SUB. & $h_{1}$ & $\{0.422, \ldots, 0.762\}$ & 0.508 \\
THICKNESS: MIDDLE SUB. & $h_{2}$ & $\{0.200\}$ & 0.200 \\
THICKNESS: TOP SUB. & $h_{3}$ & $\{0.101, \ldots, 0.508\}$ & 0.338 \\
\hline
\end{tabular}

S-parameters are measured with the PNA vector network analyzer and the radiation patterns are measured in the far-field anechoic chamber. Fig. 9 shows the radiation pattern measurement setup. The under-test antennas are mounted on a rotational plate, which covers $240^{\circ}$ of the $\Theta$ plane from $-120^{\circ}$ to $120^{\circ}$. The remaining $120^{\circ}$ area cannot be measured due to the blockage of the absorber behind the antennas. Each antenna is measured with two setups. As shown in Fig. 9(a), the measurement catches the radiation pattern in YoZ plane (rotation in $\Theta$ direction) and in Fig. 9(b), the measurement catches the XoY plane. In the array measurements, the non-tested ports are terminated with $50 \Omega$ loads.

Fig. 10(a) shows the simulated and measured S-parameters and realized gain of the single antenna. The three resonances in the simulation are observed in the measurements with a slightly frequency shift to the lower band. The measured bandwidth is from $36 \mathrm{GHz}$ to $40.6 \mathrm{GHz}$ with reflection coefficients below $-8.8 \mathrm{~dB}$. The discrepancy between the simulation and measurement is mainly due to the fabrication errors at the locations of the vias. The via location uncertainty is $\pm 0.075 \mathrm{~mm}$, which is large enough to cause an obvious frequency shift. By applying the location errors on the blind vias and the SIW, the resonant frequencies become more similar to the 


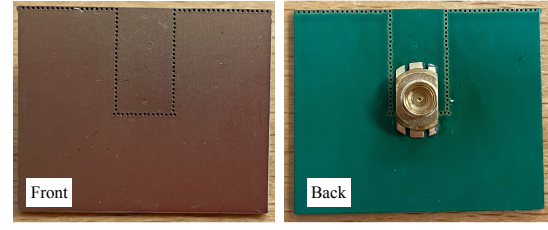

(a)
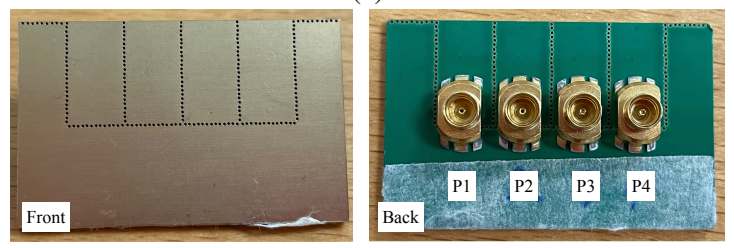

(b)

Fig. 8: Fabricated antenna and 4-element array. (a) Single antenna. (b) 4-element array.

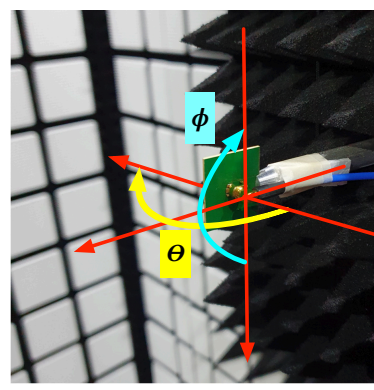

(a)

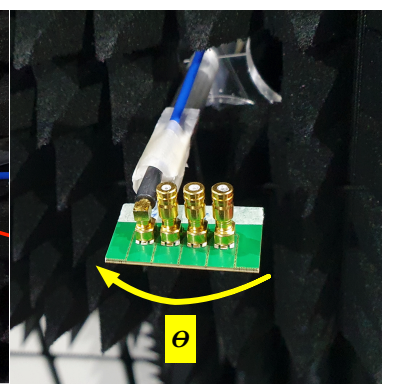

(b)

Fig. 9: Radiation pattern measurement setup. (a) measurement in YoZ plane. (b) measurement in XoY plane.

measurement but with a worse impedance matching (that may caused by other minor location errors during the fabrication). The measured realized gain ranges from $1 \mathrm{dBi}$ to $5.3 \mathrm{dBi}$ within the operating band. Correspondingly, the gain curve of the simulation considering errors is also more similar to the measurement.

The simulated and measured results of the array are shown in Fig. 10(b). Since the four array elements have very similar performances in the simulations, only $S_{11}$ and $S_{21}$ are demonstrated for comparing with the measurements. For the measurement results, the $S_{22}$ of array element $P 2$ has a slightly higher reflection compared to $S_{11}$ of array element $P 1$. The bandwidth of the array elements is from $36 \mathrm{GHz}$ to $40.7 \mathrm{GHz}$ with reflection coefficients below -8 $\mathrm{dB}$, which is similar to the result of the single antenna. The mutual coupling between array elements $P 1$ and $P 2$ is below $-22 \mathrm{~dB}$ in the measurement. The measured realized gain of array element $P 1$ and $P 2$ ranges from $1.4 \mathrm{dBi}$ to $4.9 \mathrm{dBi}$ and from $0.5 \mathrm{dBi}$ to $5.2 \mathrm{dBi}$, respectively. The results of array elements $P 3$ and $P 4$ are similar to the results of the array elements $P 1$ and $P 2$ due to the symmetry of the array. Note that these are not shown to ensure brevity.

The radiation patterns of the single antenna at $39 \mathrm{GHz}$ are shown in Fig. 11(a) and Fig. 11(b). The measured realized gain is $4.27 \mathrm{dBi}$ and the radiation pattern is in good agreement with the simulations. The simulated and measured cross-polarization levels are both below $-20 \mathrm{~dB}$. Fig. 11(c) and Fig. 11(d) are the radiation patterns of array element P2 at $39 \mathrm{GHz}$. The measured realized gain is $3.7 \mathrm{dBi}$ and the cross-polarization is below $-20 \mathrm{~dB}$.

Table III gives the comparison of the proposed antenna with the other mm-wave endfire antennas. [2] proposed a design of a quasiyagi antenna with a driven dipole and a director. [3] proposed a dipole
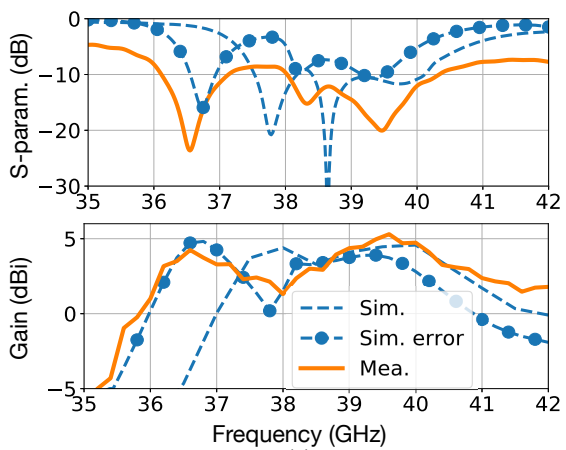

(a)
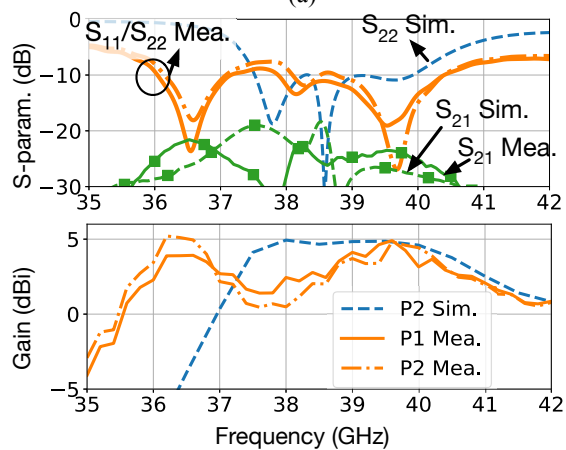

Fig. 10: Simulated and measured S-parameters and realized gain. (a) Reflection coefficients and realized gain of the single antenna. (b) Reflection coefficients, mutual coupling, and realized gain of array element P1 and P2.

antenna with the arms tilted to the ground plane in order to reduce the clearance and enhance the bandwidth (BW). References [4], [6], [10], [11] proposed SIW open-ended antennas on a thin PCB. In [4], the impedance matching is achieved by adding two symmetric metallic vias close to the SIW aperture. In [6], two rows of transition plates are adopted to improve the impedance matching. In [10], the SIW antenna has a tapered ridge inside the SIW for achieving a wideband impedance matching. In [11], the SIW open-ended antenna is air-filled fed with a tapered microstrip line obtaining a narrow band impedance matching. The antennas in the references [2]-[4], [6] and the proposed work have an inter-element distance of nearly half wavelength, which is capable of being used in a beam-steerable array for 5G mobile devices. The antennas in [10] and [11] have an wide opening width of more than $3 \lambda_{0}$. Note that the wide opening is also an important factor in improving the impedance matching and the bandwidth, but it strongly limits the scanning range. The proposed antenna has achieved zero clearance, which is the lowest compared to the other works. Besides, the proposed design has a reasonable bandwidth, which is comparable to the low-profile SIW open-ended antennas in [6] and the Yagi antenna in [2]. The proposed antenna also achieves a reasonable peak gain of $5 \mathrm{dBi}$ and a small thickness of nearly $0.1 \lambda_{0}$.

\section{CONCLUSIONS}

A novel design of a zero clearance SIW endfire antenna array for 5G mobile applications is proposed. The array element offers $12 \%$ bandwidth and a peak gain of $5.3 \mathrm{dBi}$ with zero clearance and very low profile of $1 \mathrm{~mm}\left(0.13 \lambda_{0}\right.$ at $\left.38 \mathrm{GHz}\right)$. The beam steering of the proposed array covers from $-20^{\circ}$ to $35^{\circ}$ at $39 \mathrm{GHz}$. The very compact size makes the proposed antenna very suitable for mobile applications. Moreover, the design is achieved by using a stateof-the-art machine learning-assisted antenna optimization method, 
TABLE III: Comparison of the proposed antenna against other mm-wave endfire antennas.

\begin{tabular}{|c|c|c|c|c|c|c|c|}
\hline REFERENCES & $\begin{array}{l}\text { ANTENNA } \\
\text { TYPE }\end{array}$ & $\begin{array}{c}\text { FREQUENCY } \\
(\mathrm{GHz})\end{array}$ & BANDWIDTH & $\begin{array}{l}\text { Peak Gain } \\
\quad(\mathrm{dBi})\end{array}$ & THICKNESS & Clearance & BEAM SCANNING \\
\hline Hwang, et al. (2019) [2] & YAGI & 28 & $12.3 \%$ & 5.5 & $0.07 \lambda_{0}$ & $0.23 \lambda_{0}$ & YES \\
\hline Syrytsin, et al. (2018) [3] & DIPOLE & 29 & $27.6 \%$ & 5 & $0.15 \lambda_{0}$ & $0.15 \lambda_{0}$ & YES \\
\hline $\mathrm{Li}$, et al. (2021) [4] & SIW & 27 & $18.9 \%$ & 3.4 & $0.19 \lambda_{0}$ & $0.16 \lambda_{0}$ & YES \\
\hline Lu, et al. (2020) [6] & SIW & 26 & $15.4 \%$ & 9 & $0.09 \lambda_{0}$ & $0.61 \lambda_{0}$ & YES \\
\hline Mallahzadeh, et al. (2012) [10] & SIW & 29 & $75.9 \%$ & 10 & $0.25 \lambda_{0}$ & 0 & No \\
\hline Mateo, et al. (2016) [11] & SIW & 15 & $1.4 \%$ & 8 & Not GIVEN & 0 & No \\
\hline This work & SIW & 38 & $12.0 \%$ & 5 & $0.13 \lambda_{0}$ & 0 & YES \\
\hline
\end{tabular}

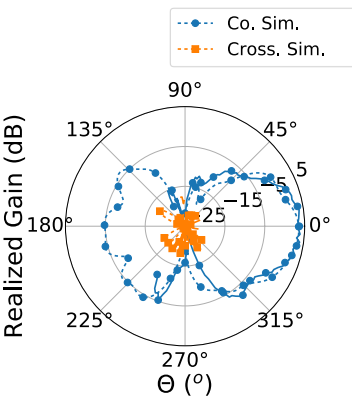

(a)

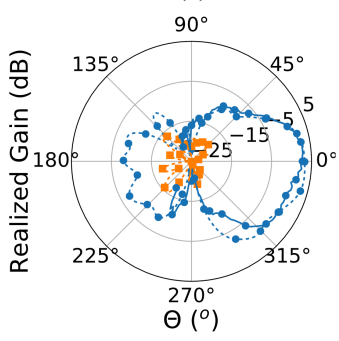

(c)

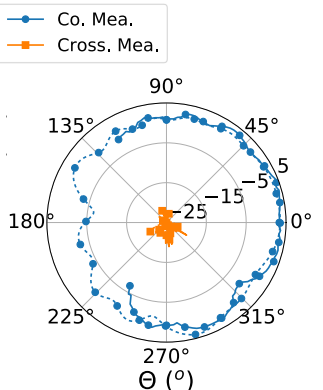

(b)

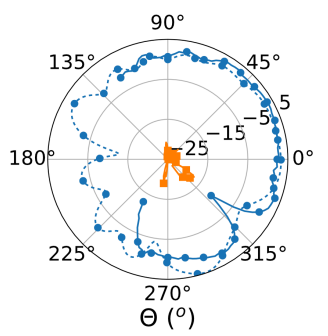

(d)

Fig. 11: Simulated and measured radiation pattern of at $39 \mathrm{GHz}$. (a) Single antenna in XoY plane. (b) Single antenna in YoZ plane. (c) Array element P2 in XoY plane. (d) Array element P2 in YoZ plane.

PSADEA. In comparison to conventional and widely used antenna optimization methods, it shows clear advantages in terms of both efficiency and design solution quality, thanks to the machine learningassisted optimization techniques. The optimization results also reveal the potential of the SIW endfire antenna in terms of the balance between the largest achievable bandwidth and the complexity of the topological profile of the structure.

\section{REFERENCES}

[1] T. S. Rappaport, S. Sun, R. Mayzus, H. Zhao, Y. Azar, K. Wang, G. N. Wong, J. K. Schulz, M. Samimi, and F. Gutierrez, "Millimeter wave mobile communications for $5 \mathrm{G}$ cellular: It will work!" IEEE Access, vol. 1, pp. 335-349, 2013.

[2] I. Hwang, B. Ahn, S. Chae, J. Yu, and W. Lee, "Quasi-yagi antenna array with modified folded dipole driver for mmwave $5 \mathrm{~g}$ cellular devices," IEEE Antennas Wireless Propag. Lett., vol. 18, no. 5, pp. 971-975, 2019.

[3] I. Syrytsin, S. Zhang, G. F. Pedersen, and A. S. Morris, "Compact quadmode planar phased array with wideband for $5 \mathrm{~g}$ mobile terminals," IEEE Trans. Antennas Propag., vol. 66, no. 9, pp. 4648-4657, 2018.

[4] H. Li, Y. Li, L. Chang, W. Sun, X. Qin, and H. Wang, "A wideband dual-polarized endfire antenna array with overlapped apertures and small clearance for 5g millimeter-wave applications," IEEE Trans. Antennas Propag., vol. 69, no. 2, pp. 815-824, 2021.

[5] M. Esquius-Morote, B. Fuchs, J. Zürcher, and J. R. Mosig, "A printed transition for matching improvement of siw horn antennas," IEEE Trans. Antennas Propag., vol. 61, no. 4, pp. 1923-1930, 2013.
[6] R. Lu, C. Yu, Y. Zhu, and W. Hong, "Compact millimeter-wave endfire dual-polarized antenna array for low-cost multibeam applications," IEEE Antennas Wireless Propag. Lett., vol. 19, no. 12, pp. 2526-2530, 2020.

[7] N. Ghassemi and K. Wu, "Planar high-gain dielectric-loaded antipodal linearly tapered slot antenna for E- and W-band gigabyte point-to-point wireless services," IEEE Trans. Antennas Propag., vol. 61, no. 4, pp. $1747-1755,2013$.

[8] Y. Cai, Y. Zhang, L. Yang, Y. Cao, and Z. Qian, "Design of low-profile metamaterial-loaded substrate integrated waveguide horn antenna and its array applications," IEEE Trans. Antennas Propag., vol. 65, no. 7, pp. 3732-3737, 2017.

[9] W. B. Park, J. M. Lee, S. Lee, Y. M. Park, and K. C. Hwang, "A 18-40 GHz substrate integrated waveguide H-plane horn antenna," IEEE Trans. Antennas Propag., vol. 66, no. 11, pp. 6322-6327, 2018.

[10] A. R. Mallahzadeh and S. Esfandiarpour, "Wideband H-plane horn antenna based on ridge substrate integrated waveguide (RSIW)," IEEE Antennas Wireless Propag. Lett., vol. 11, pp. 85-88, 2012.

[11] J. Mateo, A. M. Torres, A. Belenguer, and A. L. Borja, "Highly efficient and well-matched empty substrate integrated waveguide h-plane horn antenna," IEEE Antennas Wireless Propag. Lett., vol. 15, pp. 1510-1513, 2016.

[12] M. O. Akinsolu, B. Liu, V. Grout, P. I. Lazaridis, M. E. Mognaschi, and P. Di Barba, "A parallel surrogate model assisted evolutionary algorithm for electromagnetic design optimization," IEEE Trans. Emerg. Topics Comput., vol. 3, no. 2, pp. 93-105, 2019.

[13] B. Liu, M. O. Akinsolu, N. Ali, and R. Abd-Alhameed, "Efficient global optimisation of microwave antennas based on a parallel surrogate modelassisted evolutionary algorithm," IET Microw. Antennas Propag., vol. 13, no. 2, pp. 149-155, 2018.

[14] J. A. Tomasson, S. Koziel, and A. Pietrenko-Dabrowska, "Quasi-global optimization of antenna structures using principal components and affine subspace-spanned surrogates," IEEE Access, vol. 8, pp. 50 078-50 084, 2020.

[15] S. Koziel and A. Pietrenko-Dabrowska, "Fast multi-objective optimization of antenna structures by means of data-driven surrogates and dimensionality reduction," IEEE Access, vol. 8, pp. 183300-183311, 2020.

[16] Q. Wu, H. Wang, and W. Hong, "Multistage collaborative machine learning and its application to antenna modeling and optimization," IEEE Trans. Antennas Propag., vol. 68, no. 5, pp. 3397-3409, 2020.

[17] S. Koziel, F. Mosler, S. Reitzinger, and P. Thoma, "Robust microwave design optimization using adjoint sensitivity and trust regions," INT $J$ RF MICROW C E, vol. 22, no. 1, pp. 10-19, 2012.

[18] S. Koziel and A. Pietrenko-Dabrowska, "Reduced-cost electromagneticdriven optimisation of antenna structures by means of trust-region gradient-search with sparse jacobian updates," IET Microw. Antennas Propag., vol. 13, no. 10, pp. 1646-1652, 2019.

[19] M. Stein, "Large sample properties of simulations using latin hypercube sampling," Technometrics, pp. 143-151, 1987.

[20] J. Kennedy, Particle Swarm Optimization. Boston, MA: Springer US, 2010, pp. 760-766.

[21] Z. D. Zaharis, I. P. Gravas, T. V. Yioultsis, P. I. Lazaridis, I. A. Glover, C. Skeberis, and T. D. Xenos, "Exponential log-periodic antenna design using improved particle swarm optimization with velocity mutation," IEEE Trans. Magn., vol. 53, no. 6, pp. 1-4, 2017.

[22] B. Liu, M. O. Akinsolu, C. Song, Q. Hua, P. Excell, Q. Xu, Y. Huang, and M. A. Imran, "An efficient method for complex antenna design based on a self adaptive surrogate model-assisted optimization technique," IEEE Transactions on Antennas and Propagation, vol. 69, no. 4, pp. 2302-2315, 2021. 